\title{
Molecular cloning and expression analysis of Ganoderma boninense cyclophilins at different growth and infection stages
}

\begin{abstract}
Oil palm is subjected to various diseases including Basal Stem Rot (BSR) which is mainly caused by the basidiomycetes fungi, Ganoderma. However, studies on the fungal infection mechanism especially at molecular level and the biological processes involved are still very limited. Fungal cyclophilin (CYP) has been reported to be involved in fungal pathogenicity but involvement of CYP in the pathogenicity of Ganoderma boninense has not been revealed yet. The main objective of this study is to isolate cDNAs encoding CYP and to profile the expression level of these transcripts during infection stages of G. boninense. In this study, five full-length cDNAs encoding CYP were successfully isolated from G. boninense. They were classified as different family members of CYP because significant differences could be observed on their coding, 5' or 3' un-translated regions (UTRs). The expression of these five CYP transcripts in different type of G. boninense tissues and the infecting mycelia tissues was studied using real time quantitative PCR (qPCR). Based on the expression results and analysis, the potential functions of these CYP transcripts were predicted to be involved in the development of fruiting body (GbCYP201) and fungal stress response or pathogenicity (GbCYP203 and GbCYP205).
\end{abstract}

Keyword: Basal Stem Rot (BSR); Ganoderma boninense; Cyclophilin (CYP); Oil palm; Realtime quantitative PCR (qPCR) 